\title{
Manifestations and Outcomes of Patients with Parkinson's Disease and Serious Infection in the Emergency Department
}

\author{
Chih-Min Su, ${ }^{1,2}$ Chia-Te Kung, ${ }^{1}$ Fu-Cheng Chen $\mathbb{D}^{1},{ }^{1}$ \\ Hsien-Hung Cheng, ${ }^{1,2}$ Sheng-Yuan Hsiao, ${ }^{1,3}$ Yun-Ru Lai, ${ }^{3,4}$ Chin-Cheng Huang $\mathbb{D}^{\mathbb{D}}{ }^{4}$ \\ Nai-Wen Tsai, ${ }^{4}$ and Cheng-Hsien Lu $\mathbb{D}^{3,4,5,6}$ \\ ${ }^{1}$ Department of Emergency Medicine, Kaohsiung Chang Gung Memorial Hospital, \\ Chang Gung University College of Medicine, Kaohsiung, Taiwan \\ ${ }^{2}$ Chung Shan Medical University, School of Medicine, Taiwan \\ ${ }^{3}$ Department of Biological Science, National Sun Yat-Sen University, Kaohsiung, Taiwan \\ ${ }^{4}$ Department of Neurology, Kaohsiung Chang Gung Memorial Hospital, Chang Gung University College of Medicine, \\ Kaohsiung, Taiwan \\ ${ }^{5}$ Center for Shockwave Medicine and Tissue Engineering, Kaohsiung Chang Gung Memorial Hospital, \\ Chang Gung University College of Medicine, Kaohsiung, Taiwan \\ ${ }^{6}$ Department of Neurology, Xiamen Chang Gung Memorial Hospital, Xiamen, Fujian, China
}

Correspondence should be addressed to Cheng-Hsien Lu; chlu99@ms44.url.com.tw

Received 25 July 2018; Accepted 1 October 2018; Published 17 October 2018

Academic Editor: Gessica Sala

Copyright (C) 2018 Chih-Min Su et al. This is an open access article distributed under the Creative Commons Attribution License, which permits unrestricted use, distribution, and reproduction in any medium, provided the original work is properly cited.

\begin{abstract}
Background. Several comorbidities contribute to an increased risk of infections in Parkinson's disease (PD) as the disease progresses. However, few studies have examined the correlation between sepsis and PD. Aim. The aim of this study is to disclose the presentation and outcome of serious infection in patients with PD in the emergency department. Methods. This retrospective cohort study enrolled patients with PD who had serious infection and were admitted to the emergency department between January 2007 and December 2013. For clinical comparison, we compared the clinical features, laboratory data, and outcomes with those of age- and sex-matched patients who had serious infection but not PD. Results. There were a total of 1,200 episodes of infected PD patients and 2,400 age- and sex-matched infected patients without PD as disease controls. PD patients had fewer comorbidities and lower severity of infectious disease but longer hospital stays than control group patients. The incidences of respiratory tract and urinary tract infections were higher in PD patients. The levels of inflammatory and organ dysfunction biomarkers in PD were lower and compatible with the severity of infectious disease. A total of $86(7.2 \%)$ infected PD patients died during the 28-day admission compared to $339(14.1 \%)$ in non-PD patients. Serum C-reactive protein, bandemia, and lactate could be used to predict mortality in infected PD patients. Conclusions. In infected patients with PD, respiratory and urinary tract infections were the two most common infectious sources. Empiric therapy based on experience could treat both respiratory and urinary tract infections. Early diagnosis and treatment are essential for survival.
\end{abstract}

\section{Introduction}

Parkinson's disease (PD), the second most common neurodegenerative disease after Alzheimer's disease, includes both motor and nonmotor symptoms [1]. The features of the motor symptoms include resting tremor, rigidity, and bradykinesia [2]. As the disease progresses, hypokinesia of face muscles and a monotone change of the voice become involved and instability, rigidity worsen, and postural instability. Finally, the disease progresses to easy choking and bedridden status. Therefore, infection is a common problem in patients with advanced PD. Several nationwide studies have demonstrated that respiratory tract and urinary tract infections are the leading cause of acute emergency admission in PD patients [3-5]. Since the number of PD patients is expected to double by the year 2030, how to manage infected PD patients in the 


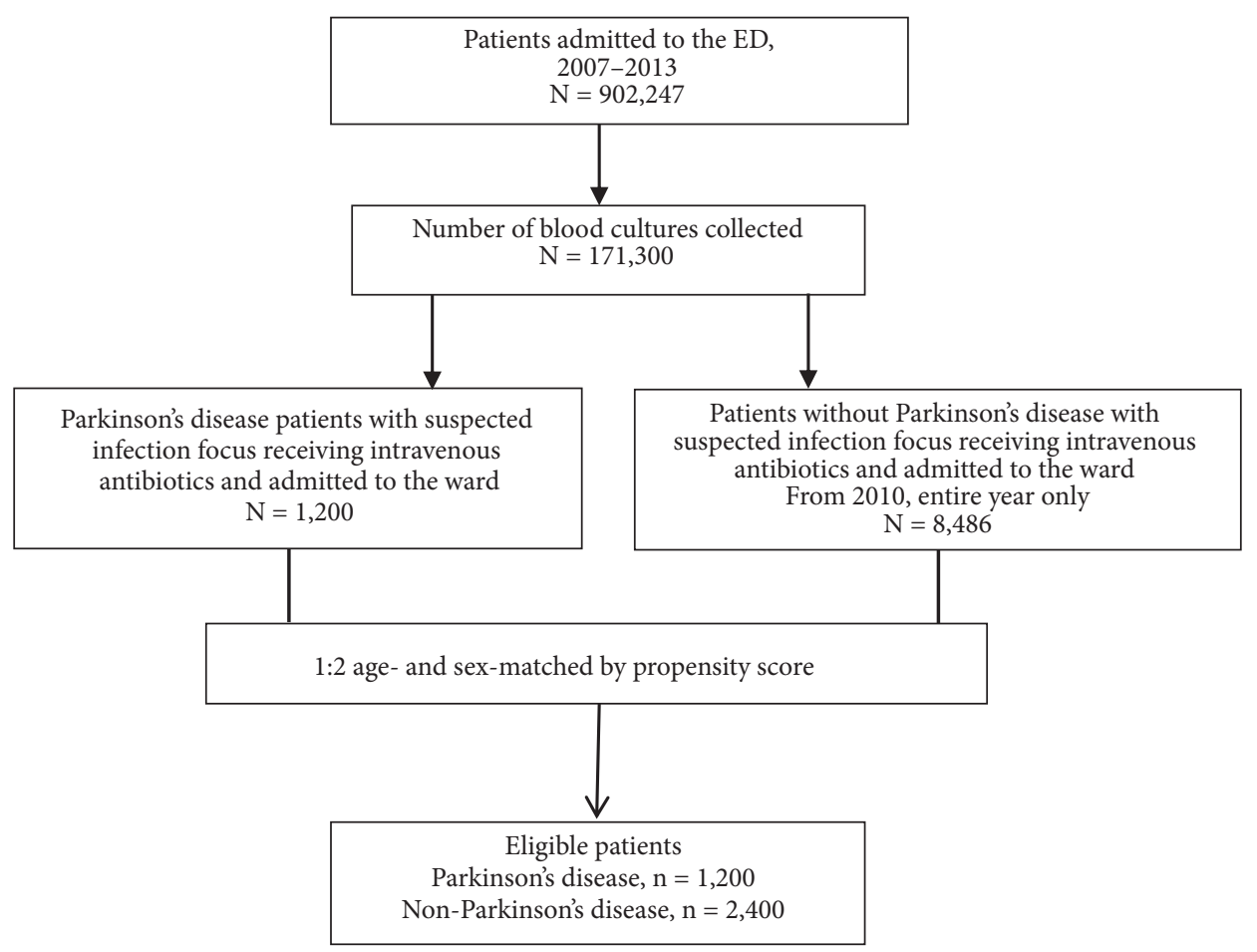

FIGURE 1: Study flow chart.

emergency department (ED) is becoming an important issue [5].

Several comorbidities (e.g., easy choking and bedridden status) contribute to the increased risk of infections in PD, but nonmotor symptomatology (e.g., autonomic dysfunction, dementia, and depression) and medications (e.g., dopaminergic or anticholinergic medications, which could cause orthostatic hypotension and mental change in elderly patients, which may mask the systemic inflammatory response) can interfere the diagnosis $[6,7]$.

Given that sepsis can be fatal, determining the clinical manifestations associated with serious infection in patients with PD could prevent delayed diagnosis. Here we aimed to analyze clinical features including infection site, laboratory data, blood culture results, hospital stay, and eventual outcome associated with serious infection. Through this study, we surveyed all infected PD patients who were admitted to the ED and compared them with age- and sex-matched infected patients to improve the therapeutic strategy.

\section{Materials and Methods}

2.1. Study Design. We conducted this single-center retrospective observational study on $\mathrm{PD}$ patients admitted to the ED with suspected serious infection, at least one set of blood culture tests, and intravenous antibiotic therapy. The data were retrieved from computerized medical records between 1 January 2007 and 31 December 2013 from Kaohsiung Chang Cheng Memorial Hospital, a 2692-bed acute-care teaching hospital, the largest medical center in Southern Taiwan providing both primary and tertiary referral care.
The institutional review board of Chang Cheng Memorial Hospital approved this study and waived the requirement for informed consent.

2.2. Study Setting and Population. All the patients from whom blood culture samples were collected in the ED were screened in a computer database as shown in Figure 1. Patients who were admitted and received intravenous antibiotics were further evaluated. All patients with PD were enrolled in the study. A ratio of 1:2 age- and sex-matched control group patients were selected by propensity score matching from the 2010 whole year patients with the same criteria. For all enrolled patients, the following data were collected retrospectively: demographic characteristics, preexisting major comorbidities, initial vital signs and laboratory tests results, major infection source, and microorganisms isolated from the blood cultures.

Blood culture samples with potential contaminating pathogens (e.g., coagulase-negative Staphylococcus, Propionibacterium acnes, Micrococcus, Corynebacterium spp., and Peptostreptococcus) were considered contaminations and were regarded as no bacteremia [8]. Laboratory data represented the result of the first test done in the ED. Septic shock is defined as sepsis-induced hypotension persisting despite adequate fluid resuscitation and inotropic agent use [9]. The major outcome was defined as 28-day in-hospital mortality.

2.3. Clinical Definitions. Since all patients were admitted for treatment, discharge diagnosis records were available for them in the database. The underlying disease and major infection focus were obtained from ICD-9 coding. PD was defined by 
TABLE 1: Demographics and clinical characteristics of septic patients with or without Parkinson's disease.

\begin{tabular}{|c|c|c|c|}
\hline Variable & $\begin{array}{c}\text { Non-PD } \\
(\mathrm{n}=2,400) \\
\end{array}$ & $\begin{array}{c}\text { PD } \\
(\mathrm{n}=1,200) \\
\end{array}$ & $P$ value \\
\hline Age, mean \pm SD & $77.3 \pm 8.8$ & $77.3 \pm 8.8$ & 0.997 \\
\hline Sex, male, n (\%) & $1313(54.7 \%)$ & $623(51.9 \%)$ & 0.119 \\
\hline \multicolumn{4}{|l|}{ Vital signs at $\mathrm{ED}($ mean $\pm \mathrm{SD})$} \\
\hline Body temperature $\left({ }^{\circ} \mathrm{C}\right)$ & $37.3 \pm 1.1$ & $37.5 \pm 1.2$ & $<0.001^{*}$ \\
\hline Heart rate (beats per minute) & $99.3 \pm 23.4$ & $98.8 \pm 21.9$ & 0.484 \\
\hline Systolic blood pressure (mmHg) & $140.4 \pm 36.8$ & $138.9 \pm 34.8$ & 0.239 \\
\hline Diastolic blood pressure (mmHg) & $80.2 \pm 29.5$ & $79.9 \pm 33.3$ & 0.834 \\
\hline Respiratory rate (breaths per minute) & $20.4 \pm 3.9$ & $20.4 \pm 3.6$ & 0.972 \\
\hline \multicolumn{4}{|l|}{ Major comorbidities, $\mathrm{n}(\%)$} \\
\hline Liver cirrhosis & $186(7.8 \%)$ & $33(2.8 \%)$ & $<0.001^{*}$ \\
\hline Hypertension & $1156(48.2 \%)$ & $605(50.4)$ & 0.203 \\
\hline Diabetes mellitus & $863(36.0 \%)$ & $443(36.9 \%)$ & 0.581 \\
\hline Chronic renal insufficiency & $247(10.3 \%)$ & $115(9.6 \%)$ & 0.518 \\
\hline Congestive heart failure & $271(11.3 \%)$ & $71(5.9 \%)$ & $<0.001^{*}$ \\
\hline Malignancy & $547(22.8 \%)$ & $114(9.5 \%)$ & $<0.001^{*}$ \\
\hline Hematologic disorder & $58(2.4 \%)$ & $9(0.8 \%)$ & $<0.001^{*}$ \\
\hline Autoimmune disease & $33(1.4 \%)$ & $7(0.6 \%)$ & $0.041^{*}$ \\
\hline \multicolumn{4}{|l|}{ Major source of infection, $\mathrm{n}(\%)$} \\
\hline Respiratory tract & $1046(43.6 \%)$ & $594(49.5 \%)$ & $0.001^{*}$ \\
\hline Urinary tract & $559(23.3 \%)$ & $583(48.6 \%)$ & $<0.001^{*}$ \\
\hline Soft tissue infection & $143(6.0 \%)$ & $87(7.2 \%)$ & 0.148 \\
\hline Meningitis & $21(0.9 \%)$ & $5(0.4 \%)$ & 0.147 \\
\hline Intra-abdominal infection & $87(3.6 \%)$ & $17(1.4 \%)$ & $<0.001^{*}$ \\
\hline Biliary tract infection & $170(7.1 \%)$ & $29(2.4 \%)$ & $<0.001^{*}$ \\
\hline Others & $656(27.3 \%)$ & $140(11.7 \%)$ & $<0.001^{*}$ \\
\hline Bacteremia, n (\%) & $331(13.8 \%)$ & $161(13.4 \%)$ & 0.797 \\
\hline Days of admission (mean \pm SD) & $15.4 \pm 13.5$ & $17.8 \pm 13.0$ & $<0.001^{*}$ \\
\hline Days of admission (survivors only) & $15.5 \pm 13.0$ & $18.1 \pm 12.9$ & $<0.001^{*}$ \\
\hline ICU admission, n (\%) & $306(12.8 \%)$ & $124(10.3 \%)$ & $0.038^{*}$ \\
\hline Septic shock within 72 hours, n (\%) & $174(7.2 \%)$ & $60(5.0 \%)$ & $0.010^{*}$ \\
\hline Respiratory failure within 72 hours, $\mathrm{n}(\%)$ & $365(15.2 \%)$ & $122(10.2 \%)$ & $<0.001^{*}$ \\
\hline 28-day in-hospital mortality, n (\%) & $339(14.1 \%)$ & $86(7.2 \%)$ & $<0.001^{*}$ \\
\hline
\end{tabular}

PD: Parkinson's disease; ED: emergency department; SD: standard deviation; ICU: intensive care unit

${ }^{*} \mathrm{P}<0.05$

specific ICD-9 coding (332.0) plus the use of Parkinsonism medication. Other comorbid underlying diseases defined by ICD-9 coding included liver cirrhosis $(571.2,571.5,571.6)$, hypertension (400.9-405.1), diabetes mellitus (250.00-250.99), congestive heart failure (428.0-428.9), renal insufficiency (582.00-589.99), malignancy (140.00-199.99), hematological disease (200.00-208.99), autoimmune disease (710.0-714.9), and stroke (430.00-438.99). Major infection focus including respiratory tract infection (481.0-486.9), urinary tract infection (590.00-590.99,601.0-601.9), biliary tract infection (576.1, 574.00-574.19, 574.30-574.49, 574.60-574.89), intraabdominal infection $(562.11,567.0-567.9)$, soft tissue infection (680.0-686.9, 728.86), and meningitis (320.00-320.99, 324.0-324.9) were defined by ICD-9 coding accordingly, while others not belonging to the above six categories were considered unknown.

2.4. Statistical Analysis. The statistical analyses were performed using the Statistical Package for the Social Sciences for Windows version 20.0 (SPSS, Chicago, IL, USA). Continuous variables are expressed as mean \pm SD and were compared using Student's t-test. Categorical variables, expressed as numbers and percentages, were compared using the $\chi^{2}$ test or Fisher's exact test. All significant variables (P values $<0.05$ ) on univariate analysis were incorporated into a hierarchical logistic regression model. We also created Cox proportional hazards model to estimate covariate-adjusted survival. P values $<0.05$ were considered statistically significant.

\section{Results}

3.1. Demographic Data of PD Patients with Serious Infection. A total of 1,200 episodes of infection events occurred in the PD patients $(\mathrm{n}=782)$ during this 7 -year period. Of them, 696 (89\%) patients had only one episode of infection, while only 12 patients (1.5\%) had $>6$ infection events. Table 1 shows that PD patients had lower percentages of liver cirrhosis $(2.8 \%$ vs. $7.8 \%, \mathrm{p}<0.001)$, congestive heart 
TABLE 2: Presentation of laboratory data of septic patients with or without Parkinson's disease.

\begin{tabular}{|c|c|c|c|}
\hline Variable & $\begin{array}{c}\text { Non-PD } \\
(\mathrm{n}=2,400)\end{array}$ & $\begin{array}{c}\text { PD } \\
(n=1,200)\end{array}$ & $\mathrm{P}$ value \\
\hline WBC $\left(1000 / \mathrm{mm}^{3}\right)$, mean \pm SD & $12.4 \pm 10.5$ & $11.9 \pm 5.4$ & $0.042^{*}$ \\
\hline Segment (\%), mean \pm SD & $78.3 \pm 13.6$ & $80.0 \pm 11.4$ & $<0.001^{*}$ \\
\hline Lymphocytes (\%), mean \pm SD & $13.0 \pm 10.2$ & $12.4 \pm 8.8$ & 0.06 \\
\hline Band form (\%), mean \pm SD & $0.9 \pm 2.7$ & $0.7 \pm 2.9$ & 0.07 \\
\hline C-reactive protein $(\mathrm{mg} / \mathrm{L})$, mean $\pm \mathrm{SD}$ & $97.0 \pm 92.2$ & $83.6 \pm 83.3$ & $<0.001^{*}$ \\
\hline Lactate $(\mathrm{mg} / \mathrm{dL})$, mean $\pm \mathrm{SD}$ & $29.8 \pm 31.8$ & $25.0 \pm 25.2$ & $0.024^{*}$ \\
\hline BUN $(\mathrm{mg} / \mathrm{dL})$, mean $\pm \mathrm{SD}$ & $31.5 \pm 28.8$ & $26.6 \pm 20.9$ & $<0.001^{*}$ \\
\hline Creatinine $(\mathrm{mg} / \mathrm{dL})$, mean $\pm \mathrm{SD}$ & $1.8 \pm 1.9$ & $1.3 \pm 1.0$ & $<0.001^{*}$ \\
\hline Bilirubin $(\mathrm{mg} / \mathrm{dL})$, mean $\pm \mathrm{SD}$ & $2.7 \pm 4.2$ & $1.3 \pm 1.3$ & $<0.001^{*}$ \\
\hline GOT $(\mathrm{U} / \mathrm{L})$, mean $\pm \mathrm{SD}$ & $107.3 \pm 503.6$ & $60.0 \pm 189.8$ & $0.009^{*}$ \\
\hline Sodium $(\mathrm{mEq} / \mathrm{L})$, mean $\pm \mathrm{SD}$ & $134.8 \pm 7.0$ & $134.5 \pm 8.0$ & 0.259 \\
\hline Potassium $(\mathrm{mEq} / \mathrm{L})$, mean $\pm \mathrm{SD}$ & $4.0 \pm 0.9$ & $4.0 \pm 0.7$ & 0.227 \\
\hline
\end{tabular}

PD: Parkinson's disease; WBC: white blood cells; SD: standard deviation; BUN: blood urea nitrogen; GOT: glutamic oxaloacetic transaminase Normal range of WBC ( 3.5 11), Segment (42-74), Lymphocyte (20-56), Band form (0-3), C-reactive protein (<5), Lactate (4.5-19.8), BUN (6-21), Creatinine (0.44-1.27), Bilirubin (0.2-1.4), GOT (0-37), Sodium (134-148), Potassium (3.5-5.2)

${ }^{*} \mathrm{P}<0.05$

failure (5.9\% vs. $11.3 \%, \mathrm{p}<0.001)$, malignancy (9.5\% vs. $22.8 \%$, $\mathrm{p}<0.001)$, hematological disease $(0.8 \%$ vs. $2.4 \%, \mathrm{p}<0.001)$, and autoimmune disease $(0.6 \%$ vs. $1.4 \%, \mathrm{p}=0.041)$ than those without PD. PD patients were at higher risk of developing respiratory tract infection $(49.5 \%$ vs. $43.6 \%, \mathrm{p}=0.001)$ and urinary tract infection $(48.6 \%$ vs. $23.3 \%, \mathrm{p}<0.001)$ but were at lower risk of developing biliary tract infection $(2.4 \%$ vs. $7.1 \%, \mathrm{p}<0.001)$ and intra-abdominal infection $(1.4 \%$ vs. $3.6 \%, \mathrm{p}<0.001)$. The indications for the severity of infection including intensive care unit (ICU) admission, septic shock, and respiratory failure all showed that PD patients were less severe than their age- and sex-matched patients. The overall in-hospital mortality rate of infected PD patients was $7.2 \%$, which was significantly lower than the $14.1 \%$ of nonPD patients $(\mathrm{p}<0.001)$. We created a multivariate regression model to predict mortality among all enrolled patients and found that PD patients had a significantly lower odds ratio (OR, 0.616; 95\% confidence interval [CI], 0.474-0.802). Although it seemed that admitted PD patients had less severe sepsis, they had longer hospital stays than overall patients (17.8 days vs. 15.4 days, $\mathrm{p}<0.001$ ) or survival-only patients (18.1 days vs. 15.5 days, $\mathrm{p}<0.001)$. Table 2 shows that the PD patients had lower levels of inflammatory and organ dysfunction biomarkers, which also inferred lower infectious disease severity. There was no difference in the percentage of bacteremia or the distribution of bacterial species between the two groups (Table 3). The leading three causative bacteria in the septic PD patients were Escherichia coli, Streptococcus spp., Klebsiella pneumoniae, and in non-PD patients were Escherichia coli, Klebsiella pneumoniae, and Staphylococcus aureus.

3.2. Risk Stratification of PD Patients with Serious Infection. Table 4 shows that patients with renal insufficiency and cancer were at a higher risk of mortality. Elderly patients with lower blood pressure at the ED were also prone to bad outcomes. Respiratory tract infection carried a higher risk of mortality than other infection sites; in contrast, urinary tract infection carried the lowest risk of mortality. After we adjusted for age and sex for all significant comorbidity and infection focus factors, we found that age (OR, 1.039; 95\% CI, 1.002-1.077), female sex (OR, 2.413; 95\% CI, 1.413-4.121), respiratory tract infection (OR, 2.451; 95\% CI, 1.365-4.403), cancer (OR, 2.804; 95\% CI, 1.468-5.536), and renal insufficiency (OR, 3.793; 95\% CI, 2.028-7.097) were significant risk factors of sepsis-related mortality, whereas urinary tract infection (OR, 0.340; 95\% CI, 0.185-0.624) carried a higher chance of survival.

3.3. Inflammatory Biomarkers in PD Patients. The presentation of infectious biomarkers among PD patients with or without mortality is shown in Table 5. Serum C-reactive protein (CRP), bandemia, and lactate levels were good indications for prediction of 28-day in-hospital mortality of infected PD patients. On receiver operating characteristic curve analysis, CRP, bandemia, and lactate had areas under the curve (AUC) of 0.648 (95\% CI, 0.575-0.721), 0.656 (95\% CI, 0.588-0.724), and 0.653 (95\% CI, 0.556-0.750), respectively, with different enrolled case numbers.

3.4. Survival Analysis of Infected PD Patients. We also performed a survival analysis of the infected PD patients. We entered age, sex, pneumonia, urinary tract infection, cancer, and renal insufficiency into the Cox regression model. It revealed that pneumonia (OR, 1.842; 95\% CI, 1.057-3.211), urinary tract infection (OR, 0.337; 95\% CI, 0.188-0.604), age (OR, 1.037; 95\% CI, 1.001-1.073), female (OR, 2.262; 95\% CI, 1.369-3.737), renal insufficiency (OR, 2.850; 95\% CI, 1.634-4.972), and cancer (OR, 2.063; 95\% CI, 1.154-3.688) had different hazard ratios in the model. Figures 2 and 3 show the survival curve by infection site categories including pneumonia and urinary tract infection. 
TABLE 3: Blood culture results for septic patients with and without Parkinson's disease.

\begin{tabular}{lcc}
\hline Bacterial strain & $\begin{array}{c}\text { Non-PD } \\
(\mathrm{n}=2,400)\end{array}$ & $\begin{array}{c}\text { PD } \\
(\mathrm{n}=1,200)\end{array}$ \\
\hline Gram-negative & $208(8.7 \%)$ & $103(8.6 \%)$ \\
Escherichia coli & $115(4.8 \%)$ & $60(5.0 \%)$ \\
Klebsiella pneumoniae & $39(1.6 \%)$ & $15(1.3 \%)$ \\
Proteus mirabilis & $5(0.2 \%)$ & $12(1.0 \%)$ \\
Pseudomonas aeruginosa & $8(0.3 \%)$ & $3(0.3 \%)$ \\
Salmonella enterica & $5(0.2 \%)$ & $1(0.1 \%)$ \\
Other & $5(0.2 \%)$ & $12(1.0 \%)$ \\
Gram-positive & $111(4.6 \%)$ & $57(4.8 \%)$ \\
Staphylococcus aureus & $35(1.5 \%)$ & $14(1.2 \%)$ \\
Staphylococcus spp. & $32(1.3 \%)$ & $12(1.0 \%)$ \\
Streptococcus spp. & $34(1.4 \%)$ & $27(2.3 \%)$ \\
Streptococcus pneumonia & $3(0.1 \%)$ & $2(0.2 \%)$ \\
Other & $8(0.3 \%)$ & $9(0.8 \%)$ \\
Anaerobic bacterium & $11(0.5 \%)$ & $0(0 \%)$ \\
Fungus & $1(0.0 \%)$ & $1(0.1 \%)$ \\
\hline
\end{tabular}

PD: Parkinson's disease

TABLE 4: Comparison of survivors and nonsurvivors of septic Parkinson's disease within 28 days of admission.

\begin{tabular}{|c|c|c|c|}
\hline Variable & $\begin{array}{l}\text { Survivors } \\
(\mathrm{n}=1,114)\end{array}$ & $\begin{array}{c}\text { Nonsurvivors } \\
(\mathrm{n}=86)\end{array}$ & P value \\
\hline Age (years), mean $\pm \mathrm{SD}$ & $77.1 \pm 8.9$ & $79.6 \pm 7.0$ & $0.011^{*}$ \\
\hline Sex, male, n (\%) & $589(52.9 \%)$ & $34(39.5 \%)$ & $0.017^{*}$ \\
\hline \multicolumn{4}{|l|}{ Vital signs at $\mathrm{ED}$, mean $\pm \mathrm{SD}$} \\
\hline Body temperature $\left({ }^{\circ} \mathrm{C}\right)$, mean $\pm \mathrm{SD}$ & $37.5 \pm 1.2$ & $37.3 \pm 1.4$ & 0.167 \\
\hline Heart rate (beats per minute), mean \pm SD & $98.5 \pm 20.9$ & $102.9 \pm 32.1$ & 0.216 \\
\hline Systolic blood pressure $(\mathrm{mmHg})$, mean $\pm \mathrm{SD}$ & $139.9 \pm 34.0$ & $125.0 \pm 41.8$ & $0.002^{*}$ \\
\hline Diastolic blood pressure $(\mathrm{mmHg})$, mean $\pm \mathrm{SD}$ & $80.6 \pm 33.8$ & $71.0 \pm 24.4$ & $0.013^{*}$ \\
\hline Respiratory rate (breaths per minute), mean \pm SD & $20.3 \pm 3.3$ & $20.7 \pm 6.1$ & 0.653 \\
\hline \multicolumn{4}{|l|}{ Major comorbidities, n (\%) } \\
\hline Liver cirrhosis & $28(2.5 \%)$ & $5(5.8 \%)$ & 0.081 \\
\hline Hypertension & $568(51.0 \%)$ & $37(43.0 \%)$ & 0.155 \\
\hline Diabetes mellitus & $421(37.8 \%)$ & $17(25.6 \%)$ & $0.024^{*}$ \\
\hline Renal insufficiency & $96(8.6 \%)$ & $19(22.1 \%)$ & $<0.001^{*}$ \\
\hline Congestive heart failure & $61(5.5 \%)$ & $10(11.6 \%)$ & $0.020^{*}$ \\
\hline Malignancy & $98(8.8 \%)$ & $16(18.6 \%)$ & $0.003^{*}$ \\
\hline Hematologic disorder & $7(0.6 \%)$ & $2(3.0 \%)$ & 0.086 \\
\hline Autoimmune disease & $7(0.6 \%)$ & $0(0 \%)$ & 1.000 \\
\hline \multicolumn{4}{|l|}{ Major source of infection, $\mathrm{n}(\%)$} \\
\hline Respiratory tract & $531(47.7 \%)$ & $63(73.3 \%)$ & $<0.001^{*}$ \\
\hline Urinary tract & $563(50.5 \%)$ & $20(23.3 \%)$ & $<0.001^{*}$ \\
\hline Soft tissue infection & $82(7.4 \%)$ & $5(5.8 \%)$ & 0.828 \\
\hline Meningitis & $4(0.4 \%)$ & $1(1.2 \%)$ & 0.265 \\
\hline Intra-abdomen infection & $17(1.5 \%)$ & $0(0 \%)$ & 0.627 \\
\hline Biliary tract infection & $27(2.4 \%)$ & $2(2.3 \%)$ & 1.000 \\
\hline Other & $126(11.3 \%)$ & $14(16.3 \%)$ & 0.167 \\
\hline Bacteremia, n (\%) & $146(13.1 \%)$ & $15(17.4 \%)$ & 0.256 \\
\hline ICU admission, n (\%) & $100(9.0 \%)$ & $24(27.9 \%)$ & $<0.001^{*}$ \\
\hline Septic shock within 72 hours, n (\%) & $41(3.7 \%)$ & $19(22.1 \%)$ & $<0.001^{*}$ \\
\hline Respiratory failure within 72 hours, $\mathrm{n}(\%)$ & $94(8.4 \%)$ & $28(32.6 \%)$ & $<0.001^{*}$ \\
\hline
\end{tabular}

SD: standard deviation; ED: emergency department; ICU: intensive care unit ${ }^{*} \mathrm{P}<0.05$ 
TABLE 5: Presentation of inflammatory and organ dysfunction biomarkers in survivors and nonsurvivors of septic PD patients within 28 days of admission.

\begin{tabular}{|c|c|c|c|c|}
\hline Biomarkers & Case numbers & Survivors & Nonsurvivors & $\mathrm{P}$ value \\
\hline WBC $\left(1000 / \mathrm{mm}^{3}\right)$, mean $\pm \mathrm{SD}$ & $1,105 / 86$ & $11.9 \pm 5.3$ & $11.9 \pm 6.4$ & 0.976 \\
\hline Segment (\%), mean \pm SD & $1,103 / 86$ & $80.2 \pm 10.8$ & $77.4 \pm 17.6$ & $0.031^{*}$ \\
\hline Lymphocyte (\%), mean \pm SD & $1,103 / 86$ & $12.4 \pm 8.4$ & $12.3 \pm 12.9$ & 0.959 \\
\hline Band form (\%), mean \pm SD & $1,103 / 86$ & $0.6 \pm 2.1$ & $3.1 \pm 7.2$ & $0.002^{*}$ \\
\hline C-reactive protein $(\mathrm{mg} / \mathrm{dL})$, mean $\pm \mathrm{SD}$ & $914 / 71$ & $79.2 \pm 78.1$ & $139.4 \pm 120.5$ & $<0.001^{*}$ \\
\hline Lactate $(\mathrm{mg} / \mathrm{dL})$, mean $\pm \mathrm{SD}$ & $220 / 38$ & $21.9 \pm 14.9$ & $43.2 \pm 52.0$ & $0.016^{*}$ \\
\hline $\mathrm{BUN}(\mathrm{mg} / \mathrm{dL})$, mean $\pm \mathrm{SD}$ & $756 / 57$ & $25.6 \pm 20.0$ & $39.8 \pm 26.7$ & $<0.001^{*}$ \\
\hline Creatinine $(\mathrm{mg} / \mathrm{dL})$, mean $\pm \mathrm{SD}$ & $1,079 / 81$ & $1.3 \pm 1.1$ & $1.5 \pm 1.0$ & $0.033^{*}$ \\
\hline Bilirubin $(\mathrm{mg} / \mathrm{dL})$, mean $\pm \mathrm{SD}$ & $182 / 16$ & $1.2 \pm 1.2$ & $1.9 \pm 2.0$ & 0.175 \\
\hline
\end{tabular}

PD: Parkinson's disease; WBC: white blood cells; SD: standard deviation; BUN: blood urea nitrogen

${ }^{*} \mathrm{P}<0.05$

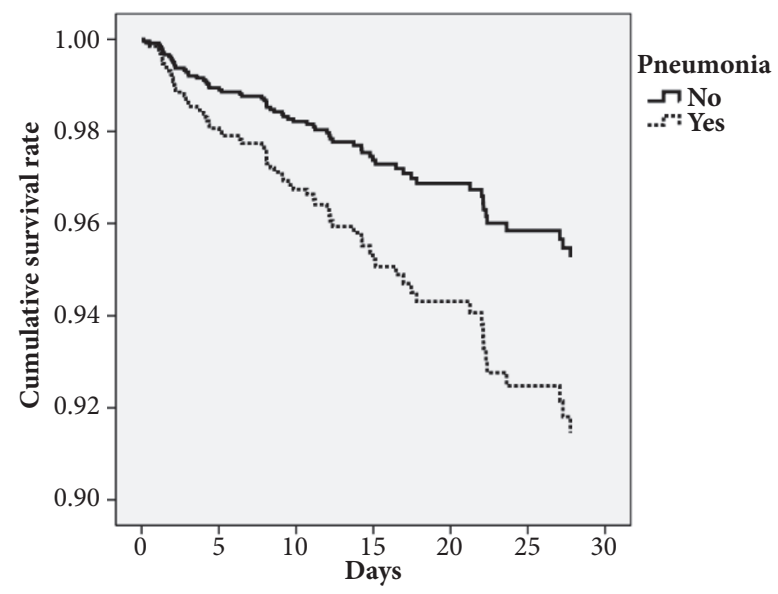

Figure 2: Survival curve of Cox regression model categorized by pneumonia status.

\section{Discussion}

To our knowledge, this is the first study to compare the clinical features and therapeutic outcomes of infected patients with or without PD. In the present study, we produced two major findings. First, the PD group was more likely to have respiratory tract and urinary tract infections than the ageand sex-matched patients. Second, although the PD group had a more benign course and lower fatality rate, they had longer mean hospitalization than the non-PD group.

The respiratory tract and the urinary tract were the two most common infection sites among PD patients in this and other studies $[5,10]$. Our study revealed that pneumonia is the leading cause of sepsis-related mortality in PD patients; in contrast, urinary tract infections had the highest survival rates. PD patients were at risk of pulmonary complications as a consequence of changes in ventilation parameters and dysphagia [11]. One study showed a link between treated dental caries and a decreased risk of pneumonia among PD patients, which suggests that aspiration pneumonia plays an important role in this group of patients [12]. The other study demonstrated that the functional dysphagia scale could be

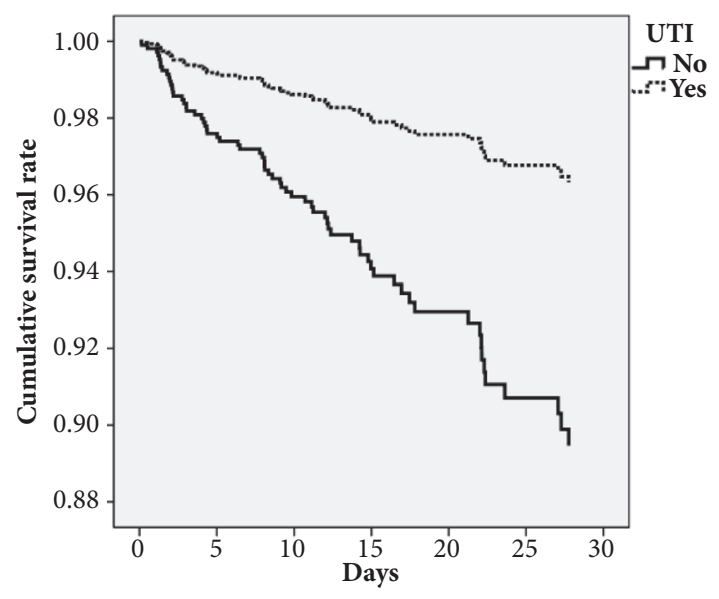

FIGURE 3: Survival curve of Cox regression model categorized by urinary tract infection status.

used to predict aspiration pneumonia in PD patients [13]. Furthermore, ventilatory dysfunction including lung volume, respiratory muscle weakness, and sleep breathing disorders were all affected in PD patients [14]. This would make PD patients more vulnerable to lung infections and unlikely to recover.

Bladder dysfunction is also a common nonmotor disorder in PD patients $[15,16]$. Symptoms include nocturia, urgency, and frequency. PD patients had problems completely emptying their bladders, which led to bacterial growth. This could explain why PD patients were more likely to have urinary tract infections than their age- and sex-matched counterparts. However, those infections were less lethal than respiratory tract infections or the other infection sites in our study.

Our data also showed that intra-abdominal infections were less common in PD patients. PD patients were susceptible to gastrointestinal problems including gastroparesis, decreased bowel movement frequency, and anorectal dysfunction [17]. However, in contrast to bladder and ventilator dysfunction, this situation did not make PD patients more vulnerable to intra-abdominal infections. 
In our study, PD patients had fewer comorbid underlying diseases than the control group, including liver cirrhosis, malignancy, congestive heart failure, and autoimmune disease. These findings are compatible with Vossius's study in which PD patients were less likely to have cardiovascular disease and cancer than non-PD patients [18]. These comorbidities would lead to higher mortality rates in the situation of sepsis and may explain the lower mortality rate in PD patients with serious infection $(7.2 \%$ vs. $14.1 \%)$. However, after we created the multivariate logistic regression model, we found that PD itself still had protective effect against sepsis-related mortality with an OR of 0.616 . Akbar also found that PD patients with aspiration pneumonia had lower mortality rates than non-PD patients (17\% vs. $22 \%)$ [19].

We found that PD patients had less fulminant clinical courses than non-PD patients demonstrated by the inflammatory and organ dysfunction biomarkers and clinical outcomes. On the other hand, although their sepsis severity was lower, PD patients had longer hospitalizations than their counterparts. Clinical studies revealed that PD patients had higher rate of frailty $[20,21]$, and it could make them look sicker and therefore seek medical help earlier and could also lead to longer hospital stay [21]. The other study also showed that PD patients had a greater chance of ED admission due to pneumonia, hip fracture, and urinary tract infection as well as longer hospital stays [4]. Our study showed that PD patients with sepsis need 2-3 more days in the hospital than sepsis patients without PD. This discrepancy showed that PD patients may need more time to recover from sepsis. Studies have shown that an inflammatory process may have harmful consequences on neurodegeneration in PD [22]. And since sepsis will provoke a pathogen-induced cytokine storm and body-wide inflammation, a certain impact on PD patients could be expected [23].

In this study, the culture rate and bacterial spectra were similar between the PD and non-PD groups. Although the culture results may not strongly represent sepsis severity or predict mortality, they could provide a clearer picture of sepsis events and guide the treatment [24]. However, as pneumonia is the most important infection source of sepsis in $\mathrm{PD}$ patients from the view of prevalence and disease severity, the positive rate of blood culture for hospitalized pneumonia in ED is around 7\%, and only half of them affected management [25]. Two-thirds of the blood cultures in PD patients were positive for gram-negative bacteria, which mainly came from the genitourinary tract and the gastrointestinal tract. Nevertheless, our data showed that, in bloodstream infections, PD patients were not different from their counterparts.

The inflammatory biomarker results demonstrated their clinical application in septic PD patients. Like in our daily practice, bandemia, serum CRP, and lactate levels could be used to diagnose sepsis or predict its severity in PD patients [26-28]. However, since PD patients have less severe sepsis than non-PD patients admitted to the ED, serum lactate levels were checked far less often in PD patients in the ED than CRP and differential white blood cell counts. The AUC of lactate for predicting 28-day sepsis-related mortality was thus lower than that of previous published data [29].
4.1. Limitations. Since this was a retrospective study, the clinical features including disease duration, PD severity (e.g., Unified Parkinson's Disease Rating Scale and Hoehn and Yahr staging scores), and daily dose of anti-Parkinsonian agents (equivalent dose of levodopa) could not be obtained from the computerized database, although infections often occurred in patients with advanced PD.

\section{Conclusions}

Sepsis occurred in PD patients, and respiratory tract and urinary tract infections were the two most common infectious sources. Empiric therapy should be based on experience and cover both respiratory tract and urinary tract infections. Although the clinical courses were more benign in septic patients with PD, they had longer hospitalizations than their non-PD counterparts. Early diagnosis and treatment are essential for survival.

\section{Data Availability}

The data used to support the findings of this study are available from the corresponding author upon request.

\section{Ethical Approval}

The study was approved by Chang Gung Memorial Hospital's Institutional Review Committee on Human Research.

\section{Conflicts of Interest}

The authors declare that there are no conflicts of interest regarding the publication of this article.

\section{References}

[1] R. B. Postuma, D. Berg, C. H. Adler et al., "The new definition and diagnostic criteria of Parkinson's disease," The Lancet Neurology, vol. 15, no. 6, pp. 546-548, 2016.

[2] R. B. Postuma, D. Berg, M. Stern et al., "MDS clinical diagnostic criteria for Parkinson's disease," Movement Disorders, vol. 30, no. 12, pp. 1591-1601, 2015.

[3] B. Kelly, C. Blake, and O. Lennon, "Acute Hospital Admissions of Individuals with a Known Parkinson's Disease Diagnosis in Ireland 2009-2012: A Short Report," Journal of Parkinson's Disease, vol. 6, no. 4, pp. 709-716, 2016.

[4] V. Low, Y. Ben-Shlomo, E. Coward, S. Fletcher, R. Walker, and C. E. Clarke, "Measuring the burden and mortality of hospitalisation in Parkinson's disease: A cross-sectional analysis of the English Hospital Episodes Statistics database 2009-2013," Parkinsonism \& Related Disorders, vol. 21, no. 5, pp. 449-454, 2015.

[5] A. Mahajan, P. Balakrishnan, A. Patel et al., "Epidemiology of inpatient stay in Parkinson's disease in the United States: Insights from the Nationwide Inpatient Sample," Journal of Clinical Neuroscience, vol. 31, pp. 162-165, 2016.

[6] K. Seppi, D. Weintraub, M. Coelho et al., "The movement disorder society evidence-based medicine review update: treatments for the non-motor symptoms of Parkinson's disease," Movement Disorders, vol. 26, no. 3, pp. S42-S80, 2011. 
[7] S. H. Fox, R. Katzenschlager, S.-Y. Lim et al., "International Parkinson and movement disorder society evidence-based medicine review: Update on treatments for the motor symptoms of Parkinson's disease," Movement Disorders, vol. 33, no. 8, pp. 1248-1266, 2018.

[8] H.-C. Chen, W.-L. Lin, C.-C. Lin et al., "Outcome of inadequate empirical antibiotic therapy in emergency department patients with community-onset bloodstream infections," Journal of Antimicrobial Chemotherapy, vol. 68, no. 4, Article ID dks475, pp. 947-953, 2013.

[9] R. P. Dellinger, M. M. Levy, A. Rhodes et al., "Surviving sepsis campaign: international guidelines for management of severe sepsis and septic shock: 2012," Intensive Care Medicine, vol. 41, no. 2, pp. 580-637, 2013.

[10] G. Wang, X.-J. Li, Y.-S. Hu et al., "Mortality from Parkinson's disease in China: Findings from a five-year follow up study in Shanghai," The Canadian Journal of Neurological Sciences. Le Journal Canadien des Sciences Neurologiques, vol. 42, no. 4, pp. 242-247, 2015.

[11] H. Shill and M. Stacy, "Respiratory Complications of Parkinson's Disease," Seminars in Respiratory and Critical Care Medicine, vol. 23, no. 3, pp. 261-266, 2002.

[12] Y. P. Chang, C. J. Yang, K. F. Hu, A. C. Chao et al., "Risk factors for pneumonia among patients with Parkinson's disease: a Taiwan nationwide population-based study," Neuropsychiatric Disease and Treatment, vol. 12, pp. 1037-1046, 2016.

[13] J. H. Lee, K. W. Lee, S. B. Kim, S. J. Lee, S. M. Chun, and S. M. Jung, "The functional dysphagia scale is a useful tool for predicting aspiration pneumonia in patients with Parkinson disease," Annals of Rehabilitation Medicine, vol. 40, no. 3, pp. 440-446, 2016.

[14] G. Baille, A. M. De Jesus, T. Perez et al., "Ventilatory dysfunction in Parkinson's disease," Journal of Parkinson's Disease, vol. 6, no. 3, pp. 463-471, 2016.

[15] R. Sakakibara, F. Tateno, M. Kishi, Y. Tsuyuzaki, T. Uchiyama, and T. Yamamoto, "Pathophysiology of bladder dysfunction in Parkinson's disease," Neurobiology of Disease, vol. 46, no. 3, pp. 565-571, 2012.

[16] L. Yeo, R. Singh, M. Gundeti, J. M. Barua, and J. Masood, "Urinary tract dysfunction in Parkinson's disease: A review," International Urology and Nephrology, vol. 44, no. 2, pp. 415424, 2012.

[17] R. F. Pfeiffer, "Gastrointestinal dysfunction in Parkinson's disease," Parkinsonism \& Related Disorders, vol. 17, no. 1, pp. 10-15, 2011.

[18] C. Vossius, O. B. Nilsen, and J. P. Larsen, "Parkinson's disease and hospital admissions: Frequencies, diagnoses and costs," Acta Neurologica Scandinavica, vol. 121, no. 1, pp. 38-43, 2010.

[19] U. Akbar, B. Dham, Y. He et al., "Incidence and mortality trends of aspiration pneumonia in Parkinson's disease in the United States, 1979-2010," Parkinsonism \& Related Disorders, vol. 21, no. 9, pp. 1082-1086, 2015.

[20] A. H. Tan, Y. C. Hew, S. Lim et al., "Altered body composition, sarcopenia, frailty, and their clinico-biological correlates, in Parkinson's disease," Parkinsonism \& Related Disorders, 2018.

[21] D. Khandelwal, A. Goel, U. Kumar, V. Gulati, R. Narang, and A. B. Dey, "Frailty is associated with longer hospital stay and increased mortality in hospitalized older patients," The Journal of Nutrition, Health \& Aging, vol. 16, no. 8, pp. 732-735, 2012

[22] M. Dufek, M. Hamanová, J. Lokaj et al., "Serum inflammatory biomarkers in Parkinson's disease," Parkinsonism \& Related Disorders, vol. 15, no. 4, pp. 318-320, 2009.
[23] N. R. London, W. Zhu, F. A. Bozza et al., "Targeting Robo4dependent slit signaling to survive the cytokine storm in sepsis and influenza," Science Translational Medicine, vol. 2, no. 23, p. 23ra19, 2010.

[24] J. Phua, W. J. Ngerng, K. C. See et al., "Characteristics and outcomes of culture-negative versus culture-positive severe sepsis," Critical Care, vol. 17, no. 5, p. R202, 2013.

[25] M. Kennedy, D. W. Bates, S. B. Wright, R. Ruiz, R. E. Wolfe, and N. I. Shapiro, "Do Emergency Department Blood Cultures Change Practice in Patients with Pneumonia?" Annals of Emergency Medicine, vol. 46, no. 5, pp. 393-400, 2005.

[26] E. Abraham, "New definitions for sepsis and septic shock: Continuing evolution but with much still to be done," Journal of the American Medical Association, vol. 315, no. 8, pp. 757-759, 2016.

[27] D. I. Agapakis, D. Tsantilas, P. Psarris et al., "Coagulation and inflammation biomarkers may help predict the severity of community-acquired pneumonia," Respirology, vol. 15, no. 5, pp. 796-803, 2010.

[28] E. Shi, G. M. Vilke, C. J. Coyne, L. C. Oyama, and E. M. Castillo, "Clinical outcomes of ED patients with bandemia," The American Journal of Emergency Medicine, vol. 33, no. 7, pp. 876881, 2015.

[29] S. M. Lee and W. S. An, "New clinical criteria for septic shock: serum lactate level as new emerging vital sign," Journal of Thoracic Disease, vol. 8, no. 7, pp. 1388-1390, 2016. 


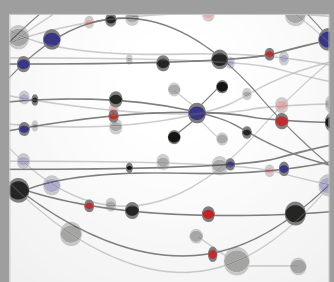

The Scientific World Journal
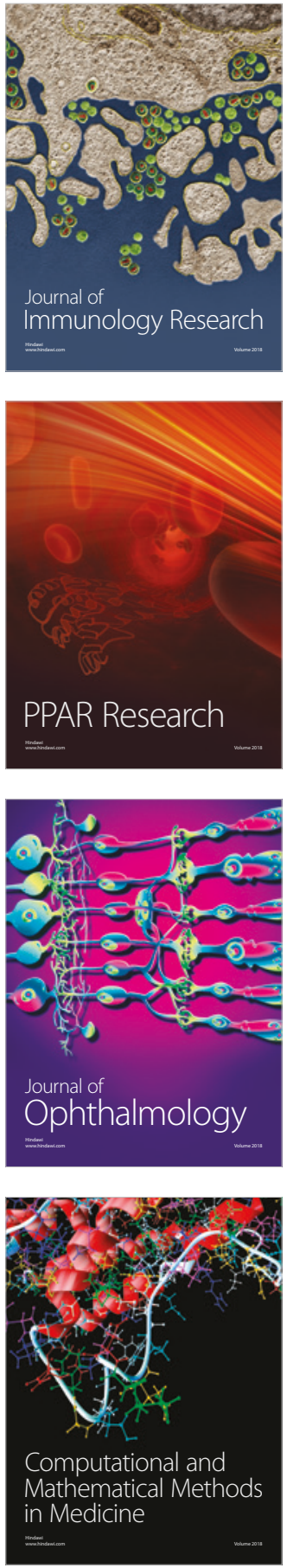

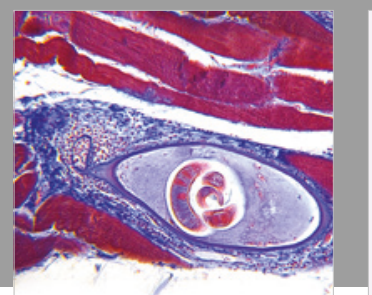

Gastroenterology Research and Practice

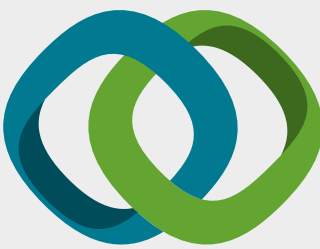

\section{Hindawi}

Submit your manuscripts at

www.hindawi.com
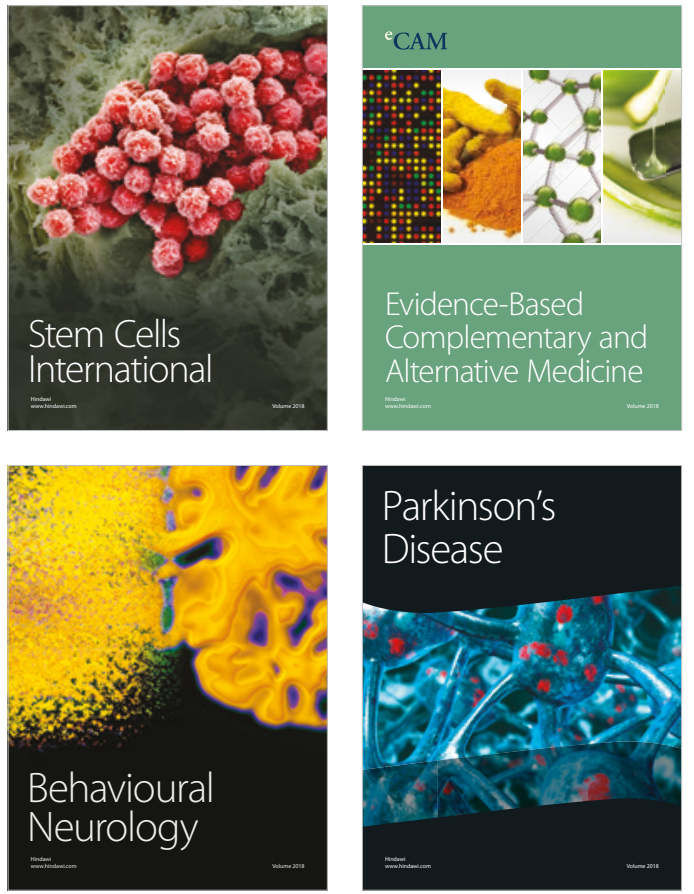

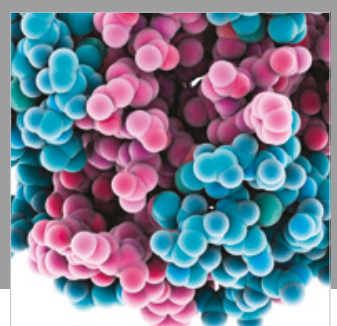

ournal of

Diabetes Research

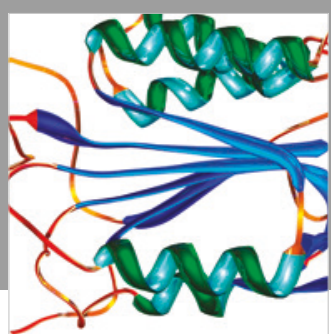

Disease Markers
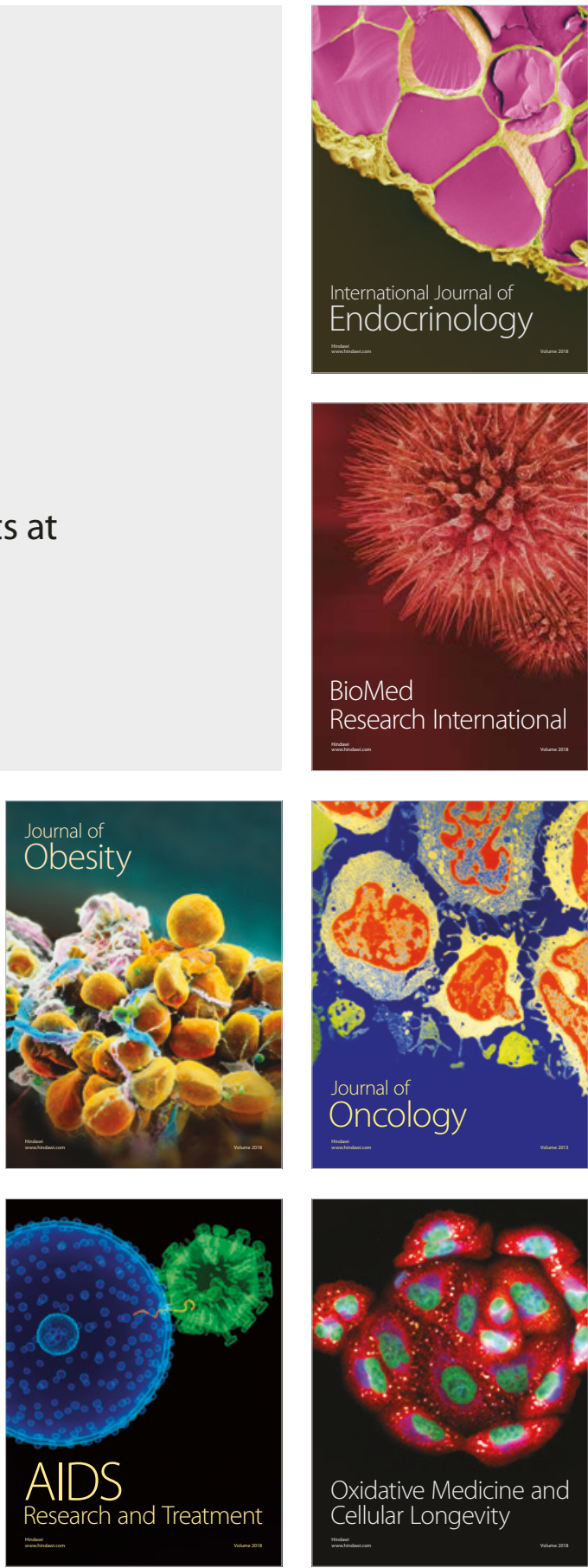